\title{
Placental infections with histologically confirmed Plasmodium falciparum are associated with adverse birth outcomes in India: a cross-sectional study
}

\author{
Rukhsana Ahmed ${ }^{1}$, Neeru Singh ${ }^{2}$, Feiko O ter Kuile ${ }^{1}$, Praveen K Bharti ${ }^{2}$, Pushpendra P Singh ${ }^{2}$, Meghna Desai ${ }^{3}$, \\ Venkatachalam Udhayakumar ${ }^{3}$ and Dianne J Terlouw ${ }^{1,4^{*}}$
}

\begin{abstract}
Background: Few studies have assessed placental malaria infections from low transmission areas by histopathology to define their impact and underlying mechanisms.

Methods: Peripheral smears and rapid diagnostic tests (RDTs), placental smears and histological samples, birth weight and gestational age were collected from 2,282 deliveries in three hospitals during a one-year (2006-2007) continuous cross-sectional survey in Madhya Pradesh. Placental histopathology included all 50 cases positive by microscopy or RDT plus 456 randomly selected samples of women negative for malaria by microscopy or RDT. Histological examination included parasites, inflammatory cells, pigment in fibrin, and morphological changes.

Results: There were 52 histology-positive cases; 38 (73.1\%) active (acute and chronic) and 14 past infections. Intervillous parasitaemia was low (60\% had $<1 \%$ parasitaemia) and monocytosis mostly mild (63\%). Compared with uninfected placentas, acute Plasmodium falciparum infections were associated with stillbirth (RR 3.8, 95\% Cl 1.2-12.1), lower maternal haemoglobin (mean difference: $1.5 \mathrm{~g} / \mathrm{dL}, 95 \% \mathrm{Cl}$ 0.5-2.5), lower birth weight (mean difference $451 \mathrm{~g}, 95 \% \mathrm{Cl}$ 169-609) and shorter gestation (mean difference 0.8 weeks, 95\% Cl 0.2-1.4). Chronic or past infections were not associated with these outcomes. Among the 11 peripheral Plasmodium vivax cases, placental parasites were absent, but they were associated with increased placental polymorphonuclear cells.
\end{abstract}

Conclusions: Malaria associated stillbirth and low birth weight in women with low protective immunity may result, at least in part, from a shortened gestation triggered by acute infection, stressing the importance of early malaria detection.

Keywords: Malaria, Pregnancy, Placenta, Histopathology, Birth weight, Haemoglobin, Gestational age, India

\section{Background}

An estimated 125 million pregnant women worldwide are exposed to the risks of malaria in pregnancy (MiP) every year, contributing to morbidity and mortality in pregnant women and newborn babies [1,2]. The pathology is related to placental sequestration due to cytoadhesion of $P$. falciparum infected erythrocytes to the placental glycosaminoglycan chondroitin sulphate A receptors and associated inflammatory responses. The resulting features of

\footnotetext{
* Correspondence: D.J.Terlouw@liverpool.ac.uk

'Department of Clinical Sciences, Liverpool School of Tropical Medicine, Liverpool, UK

${ }^{4}$ Malawi-Liverpool-Wellcome Trust Clinical Research Programme, Blantyre, Malawi

Full list of author information is available at the end of the article
}

parasite sequestration, increased presence of inflammatory cells, deposition of pigment (haemozoin), and fibrin characterize placental malaria and have been used to classify the chronology of placental infections [3,4]. The association between histologic grades of infection in P. falciparum infected placentas and low birth weight and maternal anaemia is well documented in high malaria endemic African regions [5-8], with reports of dense parasitaemia and occasional massive chronic intervillositis and monocyte infiltration mainly, but not exclusively from Africa $[9,10]$. This led to suggestions that the accumulation of parasites per se, with the corresponding inflammatory response to the sequestered parasites is associated with fetal growth restriction [11-13]. 
Reports from the Asia-Pacific and Latin America question whether the same holds for those regions. In these areas, a detrimental impact has been observed with relatively low-grade and early-pregnancy peripheral $P$. falciparum and $P$. vivax infections and placental malaria described in Colombia [14-18]. Severe manifestations of $P$. vivax infection have been associated with pronounced maternal anaemia and cytokine-mediated inflammatory response [19]. Low-grade $P$. vivax infections have been associated with intrauterine growth restriction [20], and an increased risk of miscarriage and low birth weight [21]. These findings stress the limited understanding of the population burden in areas outside Africa, and have led to recent calls for more area and species specific information to guide control strategies for these highly diverse malaria endemic regions [21].

Comprehensive, reproducible histopathology studies can help elucidate the placental changes and mechanisms that occur with $P$. falciparum and $P$. vivax infections in areas of low transmission. $P$. vivax was not considered to sequester in the microcirculation, until invitro studies recently showed that (a fraction of) $P$. vivax infected erythrocytes do have the potential to cytoadhere to the placental syncytiotrophoblast glycosaminoglycans chondroitin sulphate A responsible for $P$. falciparum sequestration in the placenta [22,23]. While this suggests a comparable pathogenesis to $P$. falciparum, the occurrence and extent of sequestration in vivo has not been shown. Three other studies recently reported histopathology findings of Plasmodium infections in Asia and Latin America [24-26], but overall the number of cases examined remain low ( $\mathrm{n}=24,19$, and 78 respectively). It is not clear how the morphological changes in the placenta described for placental malaria mainly in Africa $[7,9]$ relate to the commonly used histological grading system for placental malaria $[3,4]$, to other malaria test results and to adverse birth outcome in a low transmission setting [10].

\section{Methods}

\section{Study location}

The study sites were in three districts in Madhya Pradesh, central India. Malaria transmission in this region is highly seasonal and P. falciparum and P. vivax co-exist. In the underlying burden surveys we conducted the overall maternal malaria prevalence was $1.8 \%$ among 2282 women recruited at the time of delivery $(0.8 \%$ in the dry season and $3.3 \%$ in post rainy season). Based on standard microscopy, the prevalence of placental malaria was $1.0 \% ; 0.5 \%$ in the dry season and $2.1 \%$ in the post rainy season. P. falciparum was the predominant species in $72.5 \%$ of the observed maternal and $87.0 \%$ of placental infections.

According to a previous survey in Madhya Pradesh in $2007-8,61 \%$ of pregnant women visited ANC at least once and $34 \%$ at least three times, whereas $47.1 \%$ of women had institutional deliveries [27]. The national malaria guidelines at the time of the study recommended parasitological diagnosis with either microscopy or a rapid diagnostic malaria test (RDT) for all clinically suspected cases of malaria in pregnancy (case management), and treatment of uncomplicated $P$. vivax cases in pregnancy with chloroquine for three days, and uncomplicated $P$. falciparum cases with artesunate for three days in combination with single dose sulphadoxinepyrimethamine on day 1. Quinine was the drug of choice for first trimester treatment of $P$. falciparum. All pregnant women with anaemia (haemoglobin $[\mathrm{Hb}]<11 \mathrm{~g} / \mathrm{dL}$ ) were given haematinic supplementation (usually Fefol tablets: a combination of $150 \mathrm{mg}$ ferrous sulphate and $0.5 \mathrm{mg}$ folic acid) in the antenatal clinics. Although at the time of the study weekly chloroquine prophylaxis was recommended, this was not implemented in the participating hospitals.

\section{Procedures}

Surveys were conducted in the delivery units in a hospital in Jabalpur city between September and October 2006 and in hospitals in the towns of Katni (semi-rural) and Maihar (rural) between October 2006 and September 2007 to assess the burden of MiP. Information on demographic parameters, reproductive and malaria morbidity and prevention history during pregnancy, and delivery outcome were obtained. Newborns were weighed to the nearest 10 grams within two hours of delivery on a digital scale (Sansun, Sansui Electronics Pvt Ltd, India). Scales were calibrated weekly with a standard $5 \mathrm{~kg}$ weight. Gestational age was assessed within 24 hours of delivery using the Ballard score by staff trained by and under weekly supervision of the study paediatrician [28]. A maternal finger prick blood sample was collected during labour for malaria microscopy, pLDH-based rapid diagnostic test (RDT) (First Response $\mathrm{Pf} / \mathrm{Pv}^{\bullet}$ from Premier Medical Corporation Ltd, Mumbai, India) and haemoglobin measurement (HemoCue ${ }^{\odot}$ B-Hemoglobin, Angelholm, Sweden). All women with peripheral parasitaemia detected by microscopy or RDT were referred to the clinicians for appropriate treatment. Within three hours of delivery, placental incision and impression smears for microscopy and placental blood for PCR were taken. In addition, two $2 \times 2 \times 1 \mathrm{~cm}$ biopsies for histopathology were taken from the maternal side of the placenta at opposite sites half-way between the umbilical cord insertion and the placental edge and fixed in $10 \%$ phosphate buffered formalin. Fixed tissues were trimmed to $3 \mathrm{~mm}$ sections, placed in cassettes and fresh buffered formalin and stored in an air-conditioned room with the fixative changed monthly to ensure $\mathrm{pH}$ stability. Specimens were embedded in paraffin wax within 3 months using standard 
techniques, cut into $5 \mu \mathrm{m}$ sections, mounted on slides (two sections per placenta) and processed and stained with haematoxylin and eosin at the National Institute for Malaria Research (NIMR) field laboratory in Jabalpur, India.

Histology slides were examined locally under standard and polarized light microscopy to increase the sensitivity of haemozoin detection [29]. The reviewer (RA) was aware of the blood smear results, but unaware of the maternal characteristics, obstetric history and pregnancy outcome of the examined cases. Slides were examined under $40 \mathrm{x}$, or $100 \mathrm{x}$ magnification for the presence of parasites and inflammatory cells and scored semiquantitatively in any of the 50 fields examined. The scoring system used is presented in the Table 1 . Based on histopathology, cases with parasites were classified as active infections, consisting of chronic infections (parasites plus haemozoin in monocytes and/or fibrin) and acute infection parasites and $+/$ - pigmented monocytes with few spots or no pigmented fibrin $[3,4]$. Cases with pigment in fibrin in the absence of parasites were classified as past infections. Percentage parasitized erythrocytes was calculated against 500 maternal erythrocytes per high power (HPF, 100x magnification) and classified < $1 \%$ parasitaemia if 1-5 infected erythrocytes per HPF was observed (scanty), 1-10\% in moderate infection and $>10 \%$ parasitaemia when $>10$ infected erythrocytes was observed in 2 or more HPF (Table 1). When there was less than 500 erythrocytes per HPF the formula used was number of parasitized erythrocytes multiplied by 100 /number of maternal erythrocytes present per HPF.
PCR of placental blood was performed for species confirmation in all the 52 histology positive cases, and for an additional 63 negative cases matched for site and gravidity, selected for quality assurance for a malaria diagnosis sub-study. Placental red blood cell pellets produced at the time of placental sampling from 250-500 $\mu \mathrm{L}$ blood and stored at $-20^{\circ} \mathrm{C}$ were analysed. Nested PCR was used for genus and species specific analysis of $P$. falciparum and $P$. vivax; PCR analysis was carried out at the NIMR field station laboratory in Jabalpur [30]. The primary reaction was conducted on a volume of $25 \mu \mathrm{L}$ consisting of 5 $\mu \mathrm{L}$ DNA. Species amplification was performed on aliquot of $2 \mu \mathrm{L}$ in 1:10 dilution of first reaction mixture.

\section{Definitions}

Subpatent infections were defined as active infections detected by histopathology which were negative by any standard peripheral and placental microscopy and peripheral RDT. A low birth weight was defined as a birth weight $<2,500$ grams among singleton deliveries. Prematurity was defined as a new born delivered before the gestational age of 37 weeks among singletons as defined by the Ballard score. Fever at the time of delivery was defined as documented fever with an axillary temperature of $\geq 37.5^{\circ} \mathrm{C}$, or a history of fever in the past 24 hours at the time of delivery.

\section{Sample size}

Because of costs and labour limitations, not all placental histology samples could be processed. A sub-sample of 506 from 2,282 participants was selected for placental

Table 1 Scoring system used to evaluate presence and severity of placental pathologic changes and parasitaemia

\begin{tabular}{|c|c|c|c|}
\hline & \multicolumn{3}{|l|}{ Histopathologic severity } \\
\hline & Mild & Moderate & Severe \\
\hline Perivillous or intervillous fibrin & Present in $<20 \%$ IVS & Present in $20-50 \%$ IVS & Present in $>50 \%$ IVS \\
\hline Fibrinoid necrosis & Focal: localised to few fibrin areas & & Diffuse: $>50 \%$ fibrin affected \\
\hline Calcification & $\begin{array}{l}\text { Focal: localised to few areas } \\
\text { of fields viewed }\end{array}$ & & $\begin{array}{l}\text { Diffuse: }>50 \% \text { affected in } \\
\text { total fields viewed }\end{array}$ \\
\hline Syncytial knots & Normal: $<1 / 3$ villi affected & & $\begin{array}{l}>1 / 3 \text { villi affected in total } \\
\text { fields viewed }\end{array}$ \\
\hline Pigment deposition & Focal presence in small amounts & $\begin{array}{l}\text { Small spots or larger de- posits } \\
\text { in many locations }\end{array}$ & Large amount present widely \\
\hline Polymorphonuclear cells & $<10$ cells/HPF & 10-25 cells/HPF & $>25$ cells /HPF \\
\hline Mononuclear cells & $<10$ cells/HPF & 10-25 cells/HPF & $>25$ cells/HPF \\
\hline Parasite presence* & Scanty: 1-5/in any HPF & $5-10$ in 2 or more HPF & $>10$ in any 2 or more HPF \\
\hline Parasitaemia** & $1 \%$ & $1-10 \%$ & $>10 \%$ \\
\hline
\end{tabular}

Note: Intervillous spaces and villous structures were examined for the presence of parasitized cells and inflammatory cells. The intervillous and perivillous fibrin, inflammatory cells, villous stroma, the syncytium and Hofbauer cells were examined for the presence of pigment deposition. Morphological changes were evaluated in syncytial knots, Hofbauer cells, foetal red blood cells, villous stroma and fibrinoid necrosis and calcification. Pigment was identified as brownish granular looking material that shows birefringence under polarized light. Calcifications were identified as dark bluish purple spots that did not show birefringence on polarized light. *Plasmodium species can not be identified on histology slides, and is based on the standard microscopy and RDT results. **Percent parasitaemia is calculated against 500 erythrocytes per HPF.

Abbreviations: IVS intervillous space, HPF High power field (100x), (total fields viewed = 50), RDT rapid diagnostic tests. 
histology, including all 50 malaria positive cases $(2.2 \%$ of total sample) at delivery determined by microscopy or RDT on either peripheral, or placental smear (incision or impression smear). The remaining 456 samples were randomly selected from the remaining 2,232 smear/RDT negative women stratified by site. For every woman with a patent infection (smear or RDT positive) at least one additional infection was assumed to be detected by histology (active or past infection). A sample size of 456 smear/RDT negative cases allowed us to detect a prevalence of sub-patent infections of $\leq 3 \%$ with an absolute precision of $\pm 3 \%$ at a $95 \%$ confidence level.

\section{Statistical analysis}

Data was analysed using SAS 9.1 (Statistical Application Software, SAS Institute Inc. Cary NC). Placental changes corresponding to infection categories were evaluated using the $\chi^{2}$ test. To assess the impact of infection on birth outcomes, univariate analyses were performed using Student's $t$ test or analysis of variance (ANOVA) for continuous endpoints. Multivariate analyses were performed using General Linear Models (GLM) with birth weight, gestational age and haemoglobin as the continuous dependent variables and histology graded infection status (acute, chronic, past or no infection) as the independent predictor of interest. Additional models assessed the association between these endpoints and the individual or combination of histological parameters (parasites, syncytial knots, fibrinoid necrosis, calcification, intervillous space polymorphonuclear cells, intervillous space monocytes, intervillous space fibrin). The socio-demographic parameters; socioeconomic status, education, residence and gravidity were included in all initial models as co-variates. A significance level of $P=$ 0.05 was used to retain independent predictors in the model and 95\% confidence intervals were calculated using Tukey's multiple comparison test.

\section{Ethical approval}

The study was approved by the Research Ethical Committees of the National Institute of Malaria Research Delhi, Indian Council of Medical Research in India, CDC in U.S.A, and the Liverpool School of Tropical Medicine (LSTM) in the UK. All the study participants provided written informed consent.

\section{Results}

The mean age of women was 23.8 (3.4) years and there were $42.7 \%$ primigravidae (Table 2 ). The proportion of women reporting use of bed net the previous night was $17.8 \%$. A documented fever $(\geq 37.50 \mathrm{C})$ was present in $6.9 \%$ women and $16.2 \%$ reported fever previous week, whereas only $1.9 \%$ used anti-malarial for fever. Low birth weight was $37 \%$ and $20.8 \%$ was preterm births. In total
Table 2 Participant characteristics

\begin{tabular}{ll}
\hline Parameters & $\mathbf{N}=\mathbf{5 0 6}$ \\
\hline Age in years, mean (SD) & $23.8(3.4)$ \\
Age categories & \\
$<20 \mathrm{yr}, \mathrm{n}(\%)$ & $10(1.9)$ \\
$20-29 \mathrm{yr}, \mathrm{n}(\%)$ & $454(89.9)$ \\
$\geq 30 \mathrm{yr}, \mathrm{n}(\%)$ & $41(8.1)$
\end{tabular}

\section{Residence}

Urban, n (\%)

$148(29.2)$

Rural, n (\%)

$359(70.8)$

Education

No schooling, $\mathrm{n}(\%)$

Primary, $\mathrm{n}(\%) \quad 136(26.8)$

Secondary, $\mathrm{n}(\%) \quad 173$ (34.2)

Higher, n (\%) $36(7.1)$

$\mathrm{SES}^{1}$ rank (quintiles) $\mathbf{n}(\%)$

1 - Poorest

$117(23.1)$

2

$107(21.2)$

$112(22.1)$

85 (16.8)

$85(16.8)$

5

Gravidity

Primigravidae, n (\%) $216(42.7)$

Secundigravidae, $\mathrm{n}(\%) \quad 162(32.0)$

Gravidae-3, n (\%) $73(14.4)$

Gravidae-4, n (\%) $34(6.7)$

Multigravidae $(\geq 5)^{2} \mathrm{n}(\%) \quad 21(4.2)$

Used bednet previous night, $\mathrm{n}(\%) \quad 89$ (17.8)

ITN (any), n (\%) 1 (0.2)

Used any drug to prevent malaria, $\mathrm{n}(\%)$

Reported using haematinics, n (\%) 427 (84.7)

Morbidity and Outcomes

Reported fever previous week, n (\%) 82 (16.2)

Used antimalarial for fever, $\mathrm{n}(\%)$

Maternal fever at delivery ${ }^{3}, \mathrm{n}(\%)$

Birth weight $^{ \pm}(\mathrm{g})$, mean (SD) 2621 (450)

Birth weight $<2500 \mathrm{~g}, \mathrm{n}(\%) \quad 176$ (37.1)

Baby's gestational age in weeks ${ }^{4}$, mean, (SD) $37.2(1.3)$

Preterm babies <37 weeks), n (\%) $95(20.0)$

Stillbirths ${ }^{*}, \mathrm{n}(\%) \quad 22(4.4)$

Stillbirths in uninfected women, $\mathrm{n}(\%)$

Maternal $\mathrm{Hb}(\mathrm{g} / \mathrm{dL})$, mean (SD) 10.5 (2.2)

${ }^{1} \mathrm{SES}=$ socioeconomic status (based on underlying survey population);

$2^{2}=$ pregnancy number.

${ }^{3}=$ Fever = axillary temperature $\geq 37.5^{\circ} \mathrm{C} ;{ }^{4}=$ assessed by Ballard score; ${ }^{ \pm}$Birth weight and gestational age data was available for 474 babies out of 482 singleton live births; data for 2 babies are missing out of the 506 samples; ${ }^{*}$ Stillbirth denominator $=504$. 
there were $22(4.4 \%)$ stillbirths out of which 4 cases were in women with placental malaria.

\section{Malaria infection based on histopathology}

Placental malaria was identified in 52 of the 506 cases (Table 3). Of these, 38 (73.1\%) were active infections (20 acute infections plus 18 chronic infections) and 14 (26.9\%) were past infections. Out of the histology positive samples, 38 were positive by PCR (32 active infections and six past infections). There were 11 active infection (2.4\%) and $11(2.4 \%)$ past infections detected by placental histology that were negative by the other test techniques.

Among the 38 active infections identified by histology, 25 (65.8\%, 95\% CI 48.5-79.8) were positive by peripheral smear microscopy. These included all 20 acute infections and five of the 18 chronic infections (Table 3). All 25 infections corresponded to P. falciparum and none was due to $P$. vivax. None of the 14 cases with histological evidence of a past infection had detectable $P$. falciparum malaria by peripheral microscopy, but two were peripheral microscopy positive for $P$. vivax.

Incision smears identified 60.5\% (95\% CI 44.7-77.1) of histological P. falciparum placental infections (Table 3). The positive predictive value of an incision smear to detect $P$. falciparum placental infection was $88.5 \%$ (95\% CI 68.7-96.9) and the negative predictive value was $96.9 \%$ (94.8-98.2). Impression smears correctly identified placental infection in $44.7 \%$ (28.9-61.5) of cases, with a positive predictive value of $89.4 \%$ (65.4-98.1), and a negative

Table 3 Histopathology based placental malaria infections compared to standard microscopy and other tests

\begin{tabular}{|c|c|c|c|c|c|c|}
\hline \multirow[b]{2}{*}{ Tests } & \multicolumn{6}{|c|}{ Histopathology } \\
\hline & $\mathrm{n} / \mathrm{N}$ & $\begin{array}{l}\text { Infected* } \\
n=38\end{array}$ & $\begin{array}{l}\text { Acute } \\
n=20\end{array}$ & $\begin{array}{l}\text { Chronic } \\
n=18\end{array}$ & $\begin{array}{l}\text { Past } \\
n=14\end{array}$ & $\begin{array}{l}\text { Non-infected } \\
n=454\end{array}$ \\
\hline \multicolumn{7}{|l|}{ Any species } \\
\hline Any smear/RDT+, n (\%) & $47 / 505^{\mathrm{a}}$ & 27/38 (71.1) & 20/20 (100) & 7/18 (38.9) & $3 / 14(21.4)$ & $17 / 453(3.7)$ \\
\hline Peripheral microscopy+, n (\%) & $39 / 504^{b}$ & 25/38 (65.8) & $20 / 20(100)$ & $5 / 18(27.8)$ & $2 / 14(14.3)$ & $12 / 453(2.6)$ \\
\hline Placenta impression smear+, n (\%) & $22 / 505^{c}$ & $17 / 38(44.7)$ & $14 / 20(70.0)$ & $3 / 18(16.7)$ & $1 / 14(7.1)$ & 4/453 (0.9) \\
\hline Placental incision smear+, n (\%) & $32 / 504^{d}$ & $23 / 37(62.1)$ & $18 / 20(90.0)$ & $5 / 17(29.4)$ & $2 / 14(14.3)$ & $7 / 453(1.5)$ \\
\hline $\mathrm{RDT}+, \mathrm{n}(\%)$ & $29 / 506$ & 20/38 (52.6) & $17 / 20(85.0)$ & $3 / 18(16.7)$ & $1 / 14(7.1)$ & 8/454 (1.7) \\
\hline No infection smear/RDT, n (\%) & $458 / 505$ & 11/38 (28.9) & $0 / 20(0)$ & $11 / 18(61.1)$ & $11 / 14(78.6)$ & $436 / 453(96.2)$ \\
\hline$P C R+, n(\%)$ & $62 / 110^{\mathrm{e}}$ & $32 / 38(84.2)$ & $19 / 20(95.0)$ & $13 / 18(72.2)$ & $6 / 14(54.5)$ & $24 / 62(38.7)$ \\
\hline \multicolumn{7}{|l|}{ P. falciparum } \\
\hline Any smear/RDT+, n (\%) & $24 / 505^{\ddagger}$ & 21/38 (55.3) & $14 / 20(70.0)$ & 7/18 (38.9) & 0 & 3/453 (0.6) \\
\hline Peripheral microscopy, n (\%) & $28 / 505$ & $25 / 38(65.8)$ & $20 / 20(100)$ & $5 / 18(17.9)$ & 0 & $3 / 453(0.7)$ \\
\hline Placental impression smear, n (\%) & $19 / 505$ & $17 / 38(44.7)$ & $14 / 20(70.0)$ & $3 / 18(15.8)$ & 0 & $2 / 453(0.4)$ \\
\hline Placental incision smear, n (\%) & $26 / 504$ & 23/37 (60.5) & 18/20 (90.0) & 5/18 (19.2) & 0 & $3 / 453(0.7)$ \\
\hline RDT+, n (\%) & $12 / 506$ & $11 / 38(28.9)$ & $9 / 20(45.0)$ & 2/18 (11.1) & 0 & $1 / 454(0.2)$ \\
\hline $\mathrm{PCR}+, \mathrm{n}(\%)$ & $45 / 110^{f}$ & $30 / 38(78.9)$ & $18 / 20(90.0)$ & $12 / 18(66.6)$ & $3 / 11(21.4)$ & 12/62 (19.4 \\
\hline \multicolumn{7}{|l|}{ P. vivax } \\
\hline Any smear/RDT+, n (\%) & $14 / 505^{\neq}$ & 0 & 0 & 0 & $3 / 14(21.4)$ & $11 / 453(2.4)$ \\
\hline Peripheral microscopy, n (\%) & $11 / 505$ & 0 & 0 & 0 & $2 / 14(14.3)$ & $9 / 453(2.0)$ \\
\hline Placental impression smear, n (\%) & $3 / 504$ & 0 & 0 & 0 & $1 / 14(7.1)$ & $2 / 453(0.4)$ \\
\hline Placental incision smear, n (\%) & $6 / 505$ & 0 & 0 & 0 & $2 / 14(14.3)$ & $4 / 453(0.9)$ \\
\hline RDT+, n (\%) & $8 / 506$ & & $2 / 20(10.0)$ & $1 / 18(5.5)$ & $1 / 14(7.1)$ & 4/454 (0.8) \\
\hline$P C R+, n(\%)$ & $10 / 110^{f}$ & 0 & 0 & 0 & $3 / 14(21.4)$ & $7 / 62(11.3)$ \\
\hline \multicolumn{7}{|l|}{ Mixed infection $(P f+P v)$} \\
\hline RDT mixed +, n (\%) & $9 / 506$ & $6 / 38(15.8)$ & $6 / 20(30.0)$ & 0 & 0 & $3 / 454(0.7)$ \\
\hline PCR mixed,$+ n(\%)$ & $7 / 110$ & $2 / 38(5.3)$ & $1 / 20(5.0)$ & 1/18 (5.6) & 0 & $5 / 62(8.1)$ \\
\hline
\end{tabular}

${ }^{a}$ Out of 50 any smear/RDT positives, 3 samples were missing and were not included in the analysis; ${ }^{*}$ acute + chronic infections ${ }^{\ddagger}$ excludes mixed RDT infections ${ }^{\mathrm{b}}$ Among 39 positive peripheral smears, 11 were incision negative, and 20 were impression negative. ${ }^{\mathrm{c} A m o n g} 22$ positive impression smears, 3 were peripheral smear negative; ${ }^{d}$ Among 32 positive incision smears, 4 were peripheral smear negative; ${ }^{\text {e }}$ CR was performed for species confirmation on a sub-set of 115 samples corresponding to histology positive samples and a similar number of negative samples chosen for quality assurance matched by gravidity and study sites; fIncludes mixed species; there were no mixed species infection detected on smear microscopy. Abbreviations: Plac. = placental. 
predictive value of 95.4\% (93.0- 97.1). Combined positivity of either incision or impression smear identified $70 \%$ of histopathologically active infections.

The distribution of parasitized erythrocytes in the intervillous space was scanty: $60 \%$ showed $<1 \%$ parasitaemia and $32.5 \%$ cases had parasitaemia between 1-10\%. Individual erythrocytes were rarely seen adhering to the syncytial layer. No parasites were observed in foetal erythrocytes or within the villous structures. Among the active infections, monocyte presence was mostly mild (63\%) or moderate $(23.6 \%)$ with only 1 case of a severe intervillous infiltrate (2.6\%) (Table 4). The geometric mean parasite density of $P$. falciparum was 568 parasites $/ \mu \mathrm{L}$ and $P$. vivax was 149 parasites $/ \mu \mathrm{L}$ in maternal blood samples corresponding to the placental histology samples (not shown in the table).

\section{Histopathological changes associated with malaria}

Active and past infections were associated with an increased frequency of syncytial knotting, focal fibrinoid necrosis, focal calcification and fibrin deposits in the intervillous space (Table 4). Signs of severe infection or inflammation were not observed. The placental histological features corresponding to PCR confirmed peripheral blood mono-infections of P. falciparum $(\mathrm{n}=28)$ or P. $\operatorname{vivax}(\mathrm{n}=11)$ are presented in Table 5. Plasmodium falciparum mono-infections were more likely to be associated with mild to moderate infiltration of intervillous

Table 4 Placental histology features by category of histopathological infection (independent of Plasmodium species)

\begin{tabular}{|c|c|c|c|c|c|c|c|c|c|}
\hline Placental features & $\begin{array}{l}\mathrm{No}^{\dagger} \\
\text { Inf. } \\
\mathrm{n}=454\end{array}$ & $\begin{array}{l}\text { Past }^{+\dagger} \\
\text { Inf. } \\
n=14\end{array}$ & $\begin{array}{l}\text { Past vs } \\
\text { No inf. } \\
p \text {-value }\end{array}$ & $\begin{array}{l}\text { Active } \\
\text { Inf. } \\
n=38\end{array}$ & $\begin{array}{l}\text { Active vs } \\
\text { No inf. } \\
p \text { value }\end{array}$ & $\begin{array}{l}\text { Acute } \\
\text { Inf. } \\
n=20\end{array}$ & $\begin{array}{l}\text { Acute vs } \\
\text { No inf. } \\
p \text { value }\end{array}$ & $\begin{array}{l}\text { Chronic } \\
\text { Inf. } \\
n=18\end{array}$ & $\begin{array}{l}\text { Chronic vs } \\
\text { No inf. } \\
p \text { value }\end{array}$ \\
\hline \multicolumn{10}{|l|}{ Syncytial Knots } \\
\hline Normal, n (\%) & $414(91.2)$ & $8(57.1)$ & $<0.001$ & $18(47.4)$ & $<0.0001$ & $12(60.0)$ & 0.0003 & $6(33.3)$ & $<0.0001$ \\
\hline Increased (>1/3), n(\%) & $40(8.8)$ & $6(42.9)$ & & $20(52.6)$ & & $8(40.0)$ & & $12(66.7)$ & \\
\hline \multicolumn{10}{|l|}{ Fibrinoid necrosis } \\
\hline Absent, n (\%) & $393(86.5)$ & $7(50.0)$ & & $27(71.1)$ & & $20(100.0)$ & & $7(38.9)$ & \\
\hline Focal, n (\%) & $58(12.8)$ & $6(42.9)$ & 0.0001 & $9(23.6)$ & 0.003 & 0 & 0.15 & $9(50.0)$ & $<0.0001$ \\
\hline Diffuse, n (\%) & $3(0.7)$ & $1(7.1)$ & & $2(5.3)$ & & 0 & & $2(11.1)$ & \\
\hline \multicolumn{10}{|l|}{ Calcification } \\
\hline Absent, n (\%) & $416(91.6)$ & $9(64.3)$ & & $29(76.3)$ & & $20(100.0)$ & & $9(50.0)$ & \\
\hline Focal, n (\%) & $31(6.8)$ & $4(28.6)$ & 0.002 & 7 (18.4) & 0.008 & 0 & 0.30 & $7(38.9)$ & $<0.0001$ \\
\hline Diffuse, n (\%) & $7(1.6)$ & $1(7.1)$ & & $2(5.3)$ & & 0 & & $2(11.1)$ & \\
\hline \multicolumn{10}{|l|}{ IVS* PMN cell } \\
\hline Absent, n (\%) & $340(74.9)$ & $13(92.9)$ & & $26(70.3)$ & & $14(70.0)$ & & $12(70.6)$ & \\
\hline Mild, n (\%) & $80(17.6)$ & $1(7.1)$ & 0.47 & $9(24.3)$ & 0.75 & $4(20.0)$ & 0.93 & $5(29.4)$ & 0.45 \\
\hline Moderate, n (\%) & $33(7.3)$ & 0 & & $2(5.4)$ & & $2(10.0)$ & & 0 & \\
\hline Severe, n (\%) & $1(0.2)$ & 0 & & 0 & & 0 & & 0 & \\
\hline \multicolumn{10}{|l|}{ IVS* monocyte } \\
\hline Absent, n (\%) & $358(78.8)$ & $11(78.6)$ & & $3(7.9)$ & & $1(5.0)$ & & $2(11.1)$ & \\
\hline Mild, n (\%) & $74(16.3)$ & $3(21.4)$ & 0.63 & $24(63.2)$ & $<0.0001$ & $12(60.0)$ & $<0.0001$ & $12(66.7)$ & $<0.0001$ \\
\hline Moderate, n (\%) & $22(4.9)$ & 0 & & $10(26.3)$ & & $6(30.0)$ & & $4(22.2)$ & \\
\hline Severe, n (\%) & 0 & 0 & & $1(2.6)$ & & $1(5.0)$ & & 0 & \\
\hline \multicolumn{10}{|l|}{ IVS* fibrin } \\
\hline Absent, n (\%) & $134(29.5)$ & 0 & & $4(10.5)$ & & $4(20.0)$ & & 0 & \\
\hline Mild, n (\%) & $268(59.0)$ & $5(35.7)$ & $<0.001$ & $25(65.8)$ & 0.0002 & $15(75.0)$ & $<0.47$ & $10(55.6)$ & $<0.0001$ \\
\hline Moderate, n (\%) & $52(11.5)$ & $9(64.3)$ & & $8(21.1)$ & & $1(5.0)$ & & $7(38.9)$ & \\
\hline Severe, n (\%) & 0 & 0 & & $1(2.6)$ & & 0 & & $1(5.5)$ & \\
\hline
\end{tabular}

All are column \%. Active infection group includes 1 missing result for IVS PMN cell score. ${ }^{\dagger}$ histology classification. No-infection $=$ parasite and pigment absent, Acute infection = parasite present, \pm pigmented monocytes, \pm few spots pigment in fibrin; Chronic infection = parasite present, \pm pigmented monocytes, + pigment

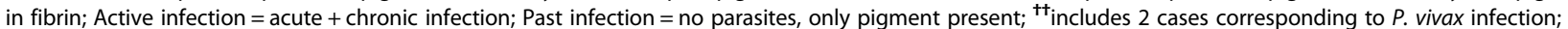
*pooled histology classified acute \& chronic infection. Abbreviations: "IVS = intervillous space; PMN = polymorphonuclear. 
Table 5 Placental histology features in peripherally patent $P$. falciparum and $P$. vivax cases

\begin{tabular}{|c|c|c|c|c|c|c|}
\hline Placental feature & $\begin{array}{l}\mathrm{No}^{\dagger} \text { infection } \\
\mathrm{n}=441\end{array}$ & $\begin{array}{l}\mathrm{Pf} \\
\mathrm{n}=\mathbf{2 8}\end{array}$ & $\begin{array}{l}\mathrm{Pv} \\
\mathrm{n}=11\end{array}$ & $\begin{array}{l}\text { Pf vs no infection } \\
P \text { value }\end{array}$ & $\begin{array}{l}P v \text { vs no Infection } \\
P \text { value }\end{array}$ & $\begin{array}{l}P f \text { vs } P v \\
P \text { value }\end{array}$ \\
\hline \multicolumn{7}{|l|}{ Syncytial Knots } \\
\hline Normal, n (\%) & $406(92.1)$ & $16(57.1)$ & $4(36.4)$ & $<0.0001$ & $<0.0001$ & 0.03 \\
\hline Increased (>1/3), n (\%) & $35(7.9)$ & $12(42.9)$ & $7(63.6)$ & & & \\
\hline \multicolumn{7}{|l|}{ Fibrinoid necrosis } \\
\hline Absent, n (\%) & $380(86.2)$ & $24(85.7)$ & $9(81.8)$ & & & \\
\hline Focal, n (\%) & $58(13.2)$ & $3(10.7)$ & $2(18.2)$ & 0.25 & 0.85 & 0.68 \\
\hline Diffuse, n (\%) & $3(0.6)$ & $1(3.6)$ & 0 & & & \\
\hline \multicolumn{7}{|c|}{ Intravillous calcification } \\
\hline Absent, n (\%) & $403(91.4)$ & $25(89.3)$ & $11(100.0)$ & & & \\
\hline Focal, n (\%) & $31(7.0)$ & $2(7.1)$ & 0 & 0.73 & 0.59 & 0.52 \\
\hline Diffuse, n (\%) & $7(1.6)$ & $1(3.6)$ & 0 & & & \\
\hline \multicolumn{7}{|l|}{ Intervillous fibrin } \\
\hline Absent, n (\%) & $125(28.3)$ & $6(21.4)$ & $7(63.6)$ & & & \\
\hline Mild, n (\%) & $265(60.1)$ & $20(71.4)$ & $4(36.4)$ & 0.48 & 0.03 & 0.03 \\
\hline Moderate, n (\%) & $51(11.6)$ & $2(7.2)$ & 0 & & & \\
\hline Severe, n (\%) & 0 & 0 & 0 & & & \\
\hline \multicolumn{7}{|c|}{ IVS polymorphonuclear cells } \\
\hline Absent, n (\%) & $335(75.9)$ & $19(70.4)$ & $3(27.3)$ & & & \\
\hline Mild, n (\%) & $77(17.5)$ & $6(22.2)$ & $3(27.3)$ & 0.91 & $<0.0001$ & 0.01 \\
\hline Moderate, n (\%) & $28(6.4)$ & $2(7.4)$ & $5(45.4)$ & & & \\
\hline Severe, n (\%) & $1(0.2)$ & 0 & 0 & & & \\
\hline \multicolumn{7}{|l|}{ Intervillous monocytes } \\
\hline Absent, n (\%) & $351(79.6)$ & $4(14.3)$ & $5(45.5)$ & & & \\
\hline Mild, n (\%) & $69(15.7)$ & $13(46.4)$ & $5(45.5)$ & $<0.0001$ & 0.02 & 0.12 \\
\hline Moderate, n (\%) & $21(4.7)$ & $10(35.7)$ & $1(9.0)$ & & & \\
\hline Severe, n (\%) & 0 & $1(3.6)$ & 0 & & & \\
\hline
\end{tabular}

All are column $\% ;{ }^{\dagger}$ negative for parasites by smear microscopy \& histology. Abbreviations: $P f=P$. falciparum, $P v=P$. vivax; IVS $=$ intervillous space.

monocytes and fibrin deposits than P. vivax monoinfection, whereas $P$. vivax infections were associated with increased syncytial knots and moderate polymorphonuclear cell infiltration.

\section{Impact of histological changes (Plasmodium falciparum) on maternal morbidity and delivery outcome}

Among the 506 cases examined for histopathology, there were 22 stillbirths. Four stillbirths occurred in women with an active infection based on histology $(4 / 38=10.5 \%)$, which was significantly higher than in uninfected women (RR 3.8, 95\% CI 1.2-12.1). Three of the four had parasitaemia $<1 \%$ (where placental microscopy identified 1-5 parasites per high power field), and one infection was classified as severe. Chronic infections were not associated with an increase in stillbirth (RR 1.3, 95\% CI 0.17-9.1).

Overall, $21.5 \%$ of women had fever (temperature $\geq$ $37.5^{\circ} \mathrm{C}$ ) at the time of delivery or history of fever in the previous week. Fever on the day of delivery was not associated with active placental infections (RR 0.8, 95\% CI $0.27-2.6)$ whereas relative risk of an active placental infection was 2.5 (95\% CI 1.5-4.0) in women with history of fever in the past week. Fever was not associated with stillbirth however; fever occurred in $27.3 \%$ [6/22] of women with a stillbirth, and $20.7 \%$ [98/473] of women with a live birth, RR 1.3, 95\% CI 0.65-2.6).

There were 482 singleton live births. The presence of parasites or pigmented monocytes was associated with decreased birth weight, gestational age at birth and maternal haemoglobin levels in univariate analysis (Table 6). In addition, increased syncytial knotting or focal calcification was associated with a shorter duration of pregnancy. Multivariate models showed that only the acute P. falciparum infections were associated with reductions in birth weight (400 g, 95\% CI 113-687) or mean gestational age (0.8 weeks, 95\% CI 0.04-1.6) or lower haemoglobin levels 
Table 6 Univariate analysis of placental Plasmodium falciparum histological determinants of birth weight, gestational age and maternal haemoglobin

\begin{tabular}{|c|c|c|c|c|c|c|c|c|c|c|c|}
\hline \multirow[b]{2}{*}{ Factors } & \multirow[b]{2}{*}{$\mathbf{N}$} & \multicolumn{3}{|c|}{ Birth weight (BW) } & \multicolumn{3}{|c|}{ Gestational age (GA) } & \multicolumn{4}{|c|}{ Maternal haemoglobin $(\mathrm{Hb})$} \\
\hline & & $\begin{array}{l}\text { Mean } \\
\text { BW (SD) }\end{array}$ & $\begin{array}{c}\text { Mean } \\
\text { difference } \\
(95 \% \mathrm{Cl})\end{array}$ & $p$ & $\begin{array}{l}\text { Mean } \\
\text { GA (SD) }\end{array}$ & $\begin{array}{c}\text { Mean } \\
\text { difference } \\
(95 \% \mathrm{Cl})\end{array}$ & $p$ & $\mathbf{N}$ & $\begin{array}{l}\text { Mean } \\
\mathrm{Hb}(\mathrm{SD})\end{array}$ & $\begin{array}{c}\text { Mean } \\
\text { difference } \\
(95 \% \mathrm{Cl})\end{array}$ & $p$ \\
\hline \multicolumn{12}{|c|}{ Placental parasites } \\
\hline Present & 33 & $2419(535)$ & $-217(-375,-58)$ & 0.01 & $36.7(1.2)$ & $-0.5(-1.03,-0.11)$ & 0.01 & 38 & $9.6(2.4)$ & $-0.9(-1.63,-0.17)$ & 0.02 \\
\hline Absent & 441 & $2636(440)$ & Reference & & $37.3(1.3)$ & Reference & & 464 & $10.5(2.1)$ & Reference & \\
\hline \multicolumn{12}{|l|}{ Syncytial knots } \\
\hline Increased & 59 & $2546(501)$ & $-85(-208,37)$ & 0.2 & $36.8(1.6)$ & $-0.5(-0.81,-0.1)$ & 0.01 & 65 & $10.3(2.6)$ & $-0.2(-0.79,0.35)$ & 0.4 \\
\hline Normal & 415 & $2631(442)$ & Reference & & $37.3(1.2)$ & Reference & & 437 & $10.5(2.1)$ & Reference & \\
\hline \multicolumn{12}{|l|}{ Fibrinoid necrosis } \\
\hline Present & 75 & $2620(476)$ & $-1(-112,110)$ & 0.9 & $37.0(1.4)$ & $-0.3(-0.58,0.05)$ & 0.1 & 78 & $10.6(2.2)$ & $0.2(-0.32,0.76)$ & 0.4 \\
\hline Absent & 399 & $2621(446)$ & Reference & & $37.3(1.3)$ & Reference & & 424 & $10.4(2.2)$ & Reference & \\
\hline \multicolumn{12}{|l|}{ Intervillous fibrin } \\
\hline Moderate-severe & 68 & $2616(432)$ & $-18(-178,141)$ & & $37.1(1.5)$ & $-0.1(-0.5,0.4)$ & & 68 & $10.2(1.8)$ & $-0.6(-1.34,0.18)$ & 0.1 \\
\hline Mild & 281 & $2616(470)$ & $-18(-133,95)$ & 0.8 & $37.2(1.3)$ & $0.0(-0.3,0.3)$ & 0.8 & 295 & $10.4(2.3)$ & $-0.4(-0.90,0.16)$ & \\
\hline Absent & 125 & $2634(417)$ & Reference & & $37.2(1.2)$ & Reference & & 139 & $10.8(2.1)$ & Reference & \\
\hline \multicolumn{12}{|l|}{ Calcification } \\
\hline Focal & 51 & 2519 (426) & $-114(-245,16)$ & 0.1 & $36.8(1.3)$ & $-0.5(-0.87,-0.11)$ & 0.01 & 51 & $10.2(2.8)$ & $-0.3(-1.03,0.24)$ & 0.2 \\
\hline Absent & 423 & $2633(452)$ & Reference & & $37.3(1.3)$ & Reference & & 451 & $10.5(2.1)$ & Reference & \\
\hline \multicolumn{12}{|l|}{ Pigmented fibrin } \\
\hline Present & 36 & $2527(452)$ & $-101(-254,52)$ & 0.2 & $37.0(1.2)$ & $-0.3(-0.72,0.16)$ & 0.2 & 40 & $10.2(2.1)$ & $-0.3(-0.98,0.44)$ & 0.4 \\
\hline Absent & 438 & $2628(450)$ & Reference & & $37.3(1.3)$ & Reference & & 462 & $10.5(2.2)$ & Reference & \\
\hline \multicolumn{12}{|c|}{ Pigmented monocytes } \\
\hline Present & 16 & $2257(516)$ & $-376(-598,-153)$ & 0.001 & $36.5(1.2)$ & $-0.7(-1.37,-0.07)$ & 0.02 & 19 & $9.1(2.7)$ & $-1.4(-2.4,-0.4)$ & 0.01 \\
\hline Absent & 458 & $2633(443)$ & Reference & & $37.3(1.3)$ & Reference & & 483 & $10.5(2.1)$ & Reference & \\
\hline \multicolumn{12}{|c|}{$\begin{array}{l}\text { *IVS Polymorponuclear } \\
\text { cells }\end{array}$} \\
\hline Moderate-severe & 33 & $2594(422)$ & $-22(-215,172)$ & & $37.5(1.1)$ & $0.3(-0.22,-0.89)$ & & 36 & $10.8(2.5)$ & $0.3(-0.54,1.26)$ & 0.5 \\
\hline Mild & 84 & $2647(436)$ & $31(-98,159)$ & 0.8 & $37.3(1.3)$ & $0.1(-0.21,0.53)$ & 0.2 & 90 & $10.4(2.3)$ & $-0.1(-0.70,0.51)$ & \\
\hline Absent & 356 & $2616(458)$ & Reference & & $37.2(1.3)$ & Reference & & 376 & $10.5(2.1)$ & Reference & \\
\hline \multicolumn{12}{|l|}{ *IVS Monocytes } \\
\hline Moderate-severe & 30 & $2634(515)$ & $-1(-202,200)$ & & $37.4(1.6)$ & $0.2(-0.42,0.74)$ & & 33 & $9.7(2.7)$ & $-0.8(-1.79,0.08)$ & \\
\hline Mild & 95 & $2563(454)$ & $-72(-195,50)$ & 0.4 & $37.1(1.2)$ & $-0.1(-0.46,0.25)$ & 0.6 & 101 & $10.5(2.4)$ & $-0.1(-0.65,0.51)$ & 0.1 \\
\hline Absent & 349 & $2635(444)$ & Reference & & $37.2(1.3)$ & Reference & & 368 & $10.6(2.1)$ & Reference & \\
\hline
\end{tabular}

Abbreviations: $\mathrm{SD}=$ standard deviation; $\mathrm{Cl}=$ confidence interval; ${ }^{*} \mathrm{IVS}=$ intervillous space; $\mathrm{BW}=$ Birth weight; $\mathrm{GA}=$ gestational age

Note: BW and GA were assessed for singleton live-birth babies only. Among 506 cases assessed, BW and GA were not available for 23 stillbirths, 1 twin delivery, and 8 newborn babies who needed immediate medical attention at birth. Maternal haemoglobin was missing in 4 of the 506 cases.

(1.4 g/dL, 95\% CI 0.5-2.4) relative to no-infection (Table 7). Further analysis showed an interaction between the effects of pigmented monocytes and parasites. The greatest effect on birth weight, gestational age and haemoglobin were observed among women who had both parasites and pigmented monocytes, whereas the effect of the presence of parasites only $(n=17)$ or of pigmented monocytes only $(\mathrm{n}=14)$ was smaller and not statistically significant (Table 7). Further stratification showed that the reduction of birth weight was greatest in women with moderate-tosevere infiltrates of pigmented monocytes, as opposed to mild levels of infiltration (Table 7).

Histopathology did not identify any active $P$. vivax infections. The presence of peripheral $P$. vivax infection (11 cases) was not associated with significant reductions in birth weight (2561 $\mathrm{g}$ [SD 200] [P. vivax, $\mathrm{n}=11]$ vs. 2639 gr [SD 445] [no P. falciparum or $P$. vivax malaria, $\mathrm{N}=445$ ], mean difference: $-78 \mathrm{~g}, 95 \% \mathrm{CI}-225.2$ to 
Table 7 Association of placental falciparum histologically-confirmed infection and birth weight, gestational age and maternal haemoglobin - multivariate analysis

\begin{tabular}{|c|c|c|c|c|c|c|c|c|c|c|c|}
\hline \multirow[b]{2}{*}{ Factors } & \multirow[b]{2}{*}{$\mathrm{N}$} & \multicolumn{3}{|c|}{ Adjusted birth weight } & \multicolumn{3}{|c|}{ Adjusted gestational age } & \multicolumn{4}{|c|}{ Adjusted maternal haemoglobin } \\
\hline & & Mean (SD) & $\begin{array}{c}\text { Mean } \\
\text { difference } \\
(95 \% \mathrm{Cl})\end{array}$ & $P$ & $\begin{array}{l}\text { Mean } \\
\text { GA (SD) }\end{array}$ & $\begin{array}{c}\text { Mean } \\
\text { difference } \\
(95 \% \mathrm{Cl})\end{array}$ & $P$ & $\mathrm{~N}$ & $\begin{array}{l}\text { Mean } \\
\text { Hb (SD) }\end{array}$ & $\begin{array}{c}\text { Mean } \\
\text { difference } \\
(95 \% \mathrm{Cl})\end{array}$ & $P$ \\
\hline \multicolumn{12}{|c|}{$\begin{array}{l}\text { 1. Infection based on } \\
\text { histopathology [3] }\end{array}$} \\
\hline Acute & 16 & $2232(541)$ & $-400(-687,-113)$ & 0.001 & $36.5(1.3)$ & $-0.8(-1.6,-0.04)$ & 0.06 & 20 & $8.8(2.4)$ & $-1.4(-2.4,-0.5)$ & 0.02 \\
\hline Chronic & 17 & $2594(481)$ & $-38(-316,240)$ & & $37.0(1.2)$ & $-0.3(-1.2,0.45)$ & & 18 & $10.6(2.2)$ & $0.1(-0.8,1.1)$ & \\
\hline Past & 12 & $2820(518)$ & $188(-141,517)$ & & $37.3(1.1)$ & $0.0(-0.9,1.0)$ & & 11 & $10.4(2.1)$ & $-0.1(-1.5,1.0)$ & \\
\hline No infection & 419 & $2632(442)$ & Reference & & $37.3(1.3)$ & Reference & & 442 & $10.5(2.1)$ & Reference & \\
\hline \multicolumn{12}{|l|}{$\begin{array}{l}\text { 2. Placental infection } \\
\text { based on }\end{array}$} \\
\hline Para $+p$ monoc & 16 & $2274(516)$ & $-356(-580,-132)$ & 0.01 & $36.6(1.2)$ & $-0.6(-1.3,0.02)$ & 0.04 & 19 & $9.3(2.7)$ & $-1.1(-2.1,-0.1)$ & 0.016 \\
\hline Parasites only & 17 & $2588(523)$ & $-42(-257,173)$ & & $36.7(1.3)$ & $-0.5(-1.2,0.07)$ & & 19 & $10.2(2.1)$ & $-0.2(-1.2,0.8)$ & \\
\hline No infection & 431 & $2630(444)$ & Reference & & $37.3(1.3)$ & Reference & & 453 & $10.4(2.2)$ & Reference & \\
\hline \multicolumn{12}{|l|}{$\begin{array}{l}\text { 3. Placental infection } \\
\text { based on }\end{array}$} \\
\hline Para + ms p monoc & 3 & $2031(582)$ & $-600(-1108,-92)$ & 0.01 & $36.2(1.0)$ & $-1.1(-2.6,0.3)$ & 0.05 & 4 & $9.1(1.5)$ & $-1.4(-3.4,0.8)$ & 0.041 \\
\hline Para + mi p monoc & 13 & $2329(507)$ & $-302(-547,-56)$ & & $36.7(1.2)$ & $-0.6(-1.2,0.2)$ & & 15 & $9.2(2.9)$ & $-1.3(-2.2,0.1)$ & \\
\hline Parasites only & 17 & $2588(523)$ & $-43(-258,173)$ & & $36.7(1.4)$ & $-0.6(-1.2,0.1)$ & & 19 & $10.2(2.2)$ & $-0.3(-1.2,0.7)$ & \\
\hline No infection & 431 & $2631(444)$ & Reference & & $37.3(1.3)$ & Reference & & 453 & $10.5(2.2)$ & Reference & \\
\hline \multicolumn{12}{|c|}{ 4. Sub-patent infections* } \\
\hline Para $+p$ monoc & 3 & $2446(490)$ & $-177(-616,263)$ & 0.4 & $37.0(0.8)$ & $-0.3(-1.6,0.9)$ & 0.8 & 4 & $10.4(4.1)$ & $-0.1(-2.1,2.1)$ & 0.9 \\
\hline Parasites only & 8 & $2778(430)$ & $155(-151,461)$ & & $37.2(1.4)$ & $-0.1(-1.0,0.8)$ & & 8 & $10.7(1.5)$ & $-0.2(-1.1,1.6)$ & \\
\hline No infection & 431 & $2623(444)$ & Reference & & $37.3(1.3)$ & Reference & & 453 & $10.5(2.1)$ & Reference & \\
\hline
\end{tabular}

$\mathrm{SD}=$ standard deviation; $\mathrm{Cl}=$ confidence interval; $P=\mathrm{p}$-value; para = parasite; $\mathrm{p}=$ pigmented; $\mathrm{mi}=$ mild; $\mathrm{ms}=$ moderate to severe; monoc $=$ monocytes. All models adjusted for socioeconomic status, gravidity, education, residence. ${ }^{*}$ Model 4 = histology positive, among those negative by any of the standard microscopy/RDT methods.

69.1), a reduced gestational age $(-0.3$ weeks, $95 \%$ CI -1.1 to 0.5$)$ or maternal haemoglobin $(-0.4 \mathrm{~g} / \mathrm{dL}, 95 \% \mathrm{CI}-1.6$ to 0.9$)$.

\section{Sub-patent placental infections (any smear or RDT negative, histology positive)}

In the 12 women who were slide smear and peripheral RDT negative but who had parasites detected by histology (sub-patent infection), the mean birth weight, gestational age and maternal haemoglobin levels were lower if both parasites and pigmented monocytes were detected, but numbers were small and the difference was not statistically significant (Table 7).

\section{Discussion}

Few studies have described placental malaria associated histopathology outside sub-Saharan Africa. Histological changes associated with $P$. falciparum and $P$. vivax infections and the corresponding impact on adverse birth outcome and maternal anaemia were assessed in an area of low malaria transmission in central India. This was a pregnant population at low risk of malaria and despite the low parasitaemia seen by the low maternal blood geometric mean parasite density and placental parasite percentage, the impact of infection was considerable and independent of the presence of documented of fever.

Based on placental incision microscopy, the overall prevalence of malaria at delivery was $1.0 \%$, and comparable to recent studies in other regions in India [31,32]. As expected, placental histology was more sensitive to detect $P$. falciparum malaria than placental or peripheral smear microscopy alone. Approximately one in 40 women (2.4\%) that were negative by conventional microscopy (either placental or peripheral) or pLDH-based RDT were found to have active placental $P$. falciparum infections (acute or chronic) by histology. When this estimate of 'subpatent' infections was extrapolated to the overall survey sample of 2282 women, the prevalence of $P$. falciparum placental malaria increased from $2.2 \%(50 / 2282)$ to $4.5 \%$, doubling the estimate. Placental incision microscopy alone missed about two out of every three infections which could be explained by the largely low parasitaemia. Women with subpatent infections had lower maternal haemoglobin, mean birth weight and gestational age, 
although the difference did not reach statistical significance, likely because of the small number of women with submicroscopic infections.

Most $(89.3 \%, 25 / 28)$ of the women with patent maternal blood $P$. falciparum infections detected by conventional microscopy also had evidence of an active placental histopathology infection. In contrast, no placental parasites could be detected by histology among the 11 women with patent peripheral $P$. vivax infections and none of the women who were smear negative for peripheral $P$. vivax had evidence of infection with histopathology. This highlights the importance of using sensitive methods such as placental histology or PCR to detect placental $P$. falciparum infection as conventional peripheral and placental microscopy smear results alone may markedly underestimate the true burden of $P$. falciparum malaria in this region.

Histopathology is a useful method to grade the chronology of placental P. falciparum infections [3,4]. Compared to observations in areas of high transmission, a relatively large proportion (38\%) of histopathological infections with $P$. falciparum were acute rather than chronic or past infections. This might be explained by the low malaria exposure and low host immunity in this population triggering an inflammatory response and early delivery when the placenta becomes infected, although the adverse impact of infection was independent of the occurrence of fever in this study. The low exposure is also reflected by the low parasitaemia within the placenta and the corresponding mild inflammatory response. This contrasts with the dense parasitaemia and the occasionally massive chronic intervillositis and monocyte infiltration reported from highly endemic areas in sub-Saharan Africa [9], but is similar to observations from the Thai-Burmese border [10] and Colombia [16-18]. Similar to previous reports, most parasites were found in the intervillous space without adhering to the syncytial layer and none were observed within the villous structure $[4,10,33]$.

Chronic P. falciparum infection was associated with an increase in syncytial knots, focal fibrinoid necrosis and calcification. These changes were also evident in past infections, suggesting they persist after parasites are cleared [4]. Alternatively, there might have been insufficient time for their resolution since time between infection and delivery might have been short, similar to observations on the Thai-Burmese border [10].

Placental P. falciparum malaria was associated with maternal anaemia. Acute infection was associated with lower levels of mean haemoglobin $(1.4 \mathrm{~g} / \mathrm{dL})$, comparable to observations in a study from Malawi [7]. Chronic infections were not associated with lower haemoglobin level. The proportion of low birth weight in this population is high and is consistent with previous reports from
India $[34,35]$. Adding to it, the impact of placental $P$. falciparum infection on birth weight was considerable, despite the scarcity of parasites within the placenta and the mild degree of placental inflammation. Of all the histological measures, only the presence of parasites and pigmented monocytic infiltration were consistently associated with reduced birth weight and shorter gestation in multivariate analysis. Further analysis showed that the effect on birth weight and gestation was particularly evident among the active infections with pigmented monocyte infiltration, a reduction in birth weight of 356 grams compared to 42 grams in women with presence of parasites alone, especially if this monocyte infiltration was moderate to severe. While mononuclear cells as a source of inflammatory cytokines have been suggested to have a role in poor foetal growth [11,36] [37-39] the infection level of 1-5 parasites per 100x magnification field approximating to $<1 \%$ parasitaemia in $60 \%$ of cases in this study also suggests that mechanisms other than the presence of placental parasitaemia per se may play a role.

The observed shortening of the duration of pregnancy with active infections was an important contributor to the reduction in birth weight. It is possible that with the increased risk of acute infections in women with history of fever, the inflammatory response to infections might induce early labour. Thus infections may not last to lead to chronicity. Alternatively clinical symptoms may lead women to seek treatment and prevent chronicity. The average duration of pregnancy was 4 days shorter among women with an active infection and mild monocyte infiltration, and 8 days shorter in women with moderate to severe infiltration. Although this may appear a relatively small difference, the average increase in birth weight between 37 to 40 weeks gestation is estimated at 169 grams per week [40]. Additionally, chronic or past infections were not associated with low birth weight or shorter gestation, which supports the notion that malaria associated low birth weight results, at least in part, from a shortening of gestation triggered by acute infections in women with low protective immunity $[4,7,9,41]$.

There was no evidence of $P$. vivax sequestration in the placenta. Pigment in the fibrin was observed in two peripherally patent $P$. vivax cases, which is consistent with reports from Thailand and Peru $[10,24]$, although previous infections with $P$. falciparum cannot be excluded. Recent studies on immune aspect of placental malaria in Colombia indicate a possible pathogenic role of $P$. vivax $[16,18]$ A noteworthy observation in Plasmodium vivax cases was the significantly higher presence of polymorphonuclear leukocytes and syncytial knots compared to $P$. falciparum infected placentas, suggesting a possible placental inflammatory response that might be different from that observed with $P$. falciparum. In addition, 
decreased fibrin deposition was seen compared to $P$. falciparum or uninfected placentas. There was no association, however, between polymorphonuclear cells or increased syncytial knots and birth weight or preterm births, although numbers may have been too small.

The study was limited by its cross-sectional design, which did not allow assessing the impact of earlier pregnancy infections via peripheral microscopy. If such infections resolve and are no longer evident as 'past' infections but still impact on maternal anaemia and birth outcome, the burden of malaria may have been underestimated. The number of women with $P$. vivax monoinfections was too low to draw firm conclusions. Although a single reader examined the sections and she was not blinded to the peripheral, placental microscopy and RDT results, a random sample of positive slides and those corresponding to patent $P$. vivax cases were reviewed blinded by a senior placental histopathologist in the US and confirmed the accuracy of the results of the first reading. PCR conducted in a subset of samples, provided further confirmation of species and the accuracy of the histological examination. The Ballard score that was used has not been validated in India. It was beyond the scope of this study to assess conditions other than malaria that may affect birth weight, haemoglobin level and prematurity (e.g. iron deficiency, other infections).

\section{Conclusions}

Severe placental inflammation associated with P. falciparum infection proved rare in this population. Despite this, a substantial impact of $P$. falciparum placental infections on birth weight was observed, which in part could be explained by shortening in the average duration of pregnancy. There was no evidence of $P$. vivax sequestration in the placenta and any histological changes observed were mild, however the number of $P$. vivax infections were small. These findings with $P$. falciparum infections highlight the need for early (including subpatent) case-detection and the challenge of malaria control during pregnancy in areas of low malaria transmission to reduce the detrimental impact of malaria during pregnancy in this region.

\section{Competing interests}

The authors declare that they have no competing interests.

\section{Authors' contributions}

RA, DJT, FTK, VU, NS and MD conceived and designed the study. RA led the overall surveys, conducted the histopathology component, data analysis and interpretation under supervision of DJT and FTK. PB and PS conducted the PCR component, RA wrote the first draft of the manuscript with input from DJT and FTK. All authors contributed to the final manuscript. All authors have contributed to, seen, and approved the final, submitted version of the manuscript.

\section{Acknowledgements}

We thank the mother baby pairs who participated in the study, the study staff for data collection, Arun Meshram and Kiran Avasti for reading the conventional smear microscopy, MP Singh for setting up the data entry screen and Pinjakar for processing the histology slides. We thank Dr Girish Majumdara at Bombay Hospital for permitting to use of their polarized light microscopy. We appreciate the cooperation received from the obstetricians, Chief Medical Officers and the antenatal and delivery unit staff of the three study hospitals and acknowledge the support of the Indian Council of Medical Research. We thank Professor Carlos Abramowsky for reviewing the selected sub-set of the histology slides, and Dr Annemieke van Eijk for her critical review of the draft. The findings and conclusions in this paper are those of the authors and do not necessarily represent the views of the Centers for Disease Control and Prevention.

\section{Author details}

'Department of Clinical Sciences, Liverpool School of Tropical Medicine, Liverpool, UK. ${ }^{2}$ National Institute of Malaria Research Field Station, Regional Medical Research Centre, Jabalpur, India. ${ }^{3}$ Malaria Branch, Division of Parasitic Diseases and Malaria, Center for Global Health, Centers for Disease Control and Prevention, Atlanta, Georgia, USA. ${ }^{4}$ Malawi-Liverpool-Wellcome Trust Clinical Research Programme, Blantyre, Malawi.

Received: 25 March 2014 Accepted: 8 June 2014

Published: 13 June 2014

\section{References}

1. Dellicour S, Tatem AJ, Guerra CA, Snow RW, ter Kuile FO: Quantifying the number of pregnancies at risk of malaria in 2007: a demographic study. PLoS Med 2010, 7:e1000221.

2. Desai M, ter Kuile FO, Nosten F, McGready R, Asamoa K, Brabin B, Newman RD: Epidemiology and burden of malaria in pregnancy. Lancet Infect Dis 2007, 7:93-104.

3. Bulmer JN, Rasheed FN, Francis N, Morrison L, Greenwood BM: Placental malaria. I. Pathological classification. Histopathology 1993, 22:211-218.

4. Ismail MR, Ordi J, Menendez C, Ventura PJ, Aponte JJ, Kahigwa E, Hirt R, Cardesa A, Alonso PL: Placental pathology in malaria: A histological, immunohistochemical, and quantitative study. Hum Pathol 2000 31:85-93.

5. Leopardi O, Naughten W, Salvia L, Colecchia M, Matteelli A, Zucchi A, Shein A, Muchi JA, Carosi G, Ghione M: Malaric placentas. A quantitative study and clinico-pathological correlations. Pathol Res Pract 1996, 192:892-898. discussion 899-900.

6. Menendez C, Ordi J, Ismail MR, Ventura PJ, Aponte JJ, Kahigwa E, Font F, Alonso PL: The impact of placental malaria on gestational age and birth weight. J Infect Dis 2000, 181:1740-1745.

7. Rogerson SJ, Pollina E, Getachew A, Tadesse E, Lema VM, Molyneux ME: Placental monocyte infiltrates in response to Plasmodium falciparum malaria infection and their association with adverse pregnancy outcomes. Am J Trop Med Hyg 2003, 68:115-119.

8. Watkinson M, Rushton Dl: Plasmodial pigmentation of placenta and outcome of pregnancy in West African mothers. BMJ 1983, 287:251-254

9. Ordi J, Ismail MR, Menendez C, Ventura PJ, Cardesa A: Massive chronic intervillositis associated with malaria infection. Modern Pathology 1998, 11:146A.

10. McGready R, Davison BB, Stepniewska K, Shee TCF, Brockman A, Udomsangpetch R, Loareesuwan S, White NJ, Meshnick SR, Nosten F: The effects of Plasmodium falciparum and P. vivax infections on placental histopathology in an area of low malaria transmission. Am J Trop Med Hyg e 2004, 70:398-407.

11. Abrams ET, Brown H, Chensue SW, Turner GD, Tadesse E, Lema VM, Molyneux ME, Rochford R, Meshnick SR, Rogerson SJ: Host response to malaria during pregnancy: placental monocyte recruitment is associated with elevated beta chemokine expression. J Immunol 2003, 170:2759-2764.

12. Duffy PE: Immunity to Malaria during Pregnancy: Different Host, Different Parasite. 1st edition. London: Taylor \& Francis; 2001

13. Muehlenbachs A, Fried M, McGready R, Harrington WE, Mutabingwa TK, Nosten F, Duffy PE: A novel histological grading scheme for placental malaria applied in areas of high and low malaria transmission. $J$ Infect Dis 2010, 202:1608-1616. 
14. Nosten F, McGready R, Thwai KL, Cho T, Hkirijaroen L, Looareesuwan S, White NJ: Effects of Plasmodium vivax malaria in pregnancy. Lancet 1999, 354:546-549.

15. McGready R, Lee SJ, Wiladphaingern J, Ashley EA, Rijken MJ, Boel M, Simpson JA, Paw MK, Pimanpanarak M, Mu O, Singhasivanon P, White NJ, Nosten FH: Adverse effects of falciparum and vivax malaria and the safety of antimalarial treatment in early pregnancy: a population-based study. Lancet Infect Dis 2012, 12:388-396.

16. Arango EM, Samuel R, Agudelo OM, Carmona-Fonseca J, Maestre A, Yanow SK: Molecular detection of malaria at delivery reveals a high frequency of submicroscopic infections and associated placental damage in pregnant women from northwest Colombia. Am J Trop Med Hyg 2013, 89:178-183.

17. Carmona-Fonseca J, Arango E, Maestre A: Placental malaria in Colombia: histopathologic findings in Plasmodium vivax and $P$. falciparum infections. Am J Trop Med Hyg 2013, 88:1093-1101.

18. Agudelo O, Arango E, Maestre A, Carmona-Fonseca J: Prevalence of gestational, placental and congenital malaria in north-west Colombia. Malar J 2013, 12:341

19. Anstey NM, Russell B, Yeo TW, Price RN: The pathophysiology of vivax malaria. Trends Parasitol 2009, 25:220-227.

20. Rijken MJ, Papageorghiou AT, Thiptharakun S, Kiricharoen S, Dwell SL, Wiladphaingern J, Pimanpanarak M, Kennedy SH, Nosten F, McGready R: Ultrasound evidence of early fetal growth restriction after maternal malaria infection. PLoS One 2012, 7:e31411.

21. Rijken MJ, McGready R, Boel ME, Poespoprodjo R, Singh N, Syafruddin D, Rogerson S, Nosten F: Malaria in pregnancy in the Asia-Pacific region. Lancet Infect Dis 2012, 12:75-88.

22. Carvalho BO, Lopes SC, Nogueira PA, Orlandi PP, Bargieri DY, Blanco YC, Mamoni R, Leite JA, Rodrigues MM, Soares IS, Oliveira TR, Wunderlich G, Lacerda MV, del Portillo HA, Araújo MO, Russell B, Suwanarusk R, Snounou G, Rénia L, Costa FT: On the cytoadhesion of Plasmodium vivax-infected erythrocytes. J Infect Dis 2010, 202:638-647.

23. Chotivanich K, Udomsangpetch $R$, Suwanarusk R, Pukrittayakamee $S$, Wilairatana P, Beeson JG, Day NP, White NJ: Plasmodium vivax adherence to placental glycosaminoglycans. PLoS One 2012, 7:e34509.

24. Parekh FK, Davison BB, Gamboa D, Hernandez J, Branch OH: Placental histopathologic changes associated with subclinical malaria infection and its impact on the fetal environment. Am J Trop Med Hyg 2010 83:973-980.

25. Mayor A, Bardaji A, Felger I, King CL, Cistero P, Dobano C, Stanisic DI, Siba P, Wahlgren M, del Portillo H, Mueller I, Menéndez C, Ordi J, Rogerson S: Placental infection with Plasmodium vivax: a histopathological and molecular study. J Infect Dis 2012, 206:1904-1910.

26. Souza RM, Ataide R, Dombrowski JG, Ippolito V, Aitken EH, Valle SN, Alvarez JM, Epiphanio S, Marinho CR: Placental histopathological changes associated with Plasmodium vivax infection during pregnancy. PLOS Negl Trop Dis 2013, 7:e2071

27. International Institute for Population Sciences: India Madhya Pradesh District Level Household and Facility Survey (DLHS-3), 2007-08. Mumbai, India: International Institute for Population Services; 2010.

28. Ballard JL, Novak KK, Driver M: A simplified score for assessment of fetal maturation of newly born infants. J Pediatr 1979, 95:769-774.

29. Romagosa C, Menendez C, Ismail MR, Quinto L, Ferrer B, Alonso PL, Ordi J: Polarisation microscopy increases the sensitivity of hemozoin and Plasmodium detection in the histological assessment of placental malaria. Acta Trop 2004, 90:277-284.

30. Snounou G, Beck HP: The use of PCR genotyping in the assessment of recrudescence or reinfection after antimalarial drug treatment. Parasitol Today 1998, 14:462-467.

31. Singh N, Singh MP, Wylie BJ, Hussain M, Kojo YA, Shekhar C, Sabin L, Desai M, Udhayakumar $V$, Hamer DH: Malaria prevalence among pregnant women in two districts with differing endemicity in Chhattisgarh. India. Malar J 2012, 11:274

32. Hamer DH, Singh MP, Wylie BJ, Yeboah-Antwi K, Tuchman J, Desai M, Udhayakumar V, Gupta P, Brooks MI, Shukla MM, Awasthy K, Sabin L, MacLeod WB, Dash AP, Singh N: Burden of malaria in pregnancy in Jharkhand State. India. Malar J 2009, 8:210.

33. Yamada M, Steketee R, Abramowsky C, Kida M, Wirima J, Heymann D, Rabbege J, Breman J, Aikawa M: Plasmodium falciparum associated placental pathology: a light and electron microscopic and immunohistologic study. Am J Trop Med Hyg 1989, 41:161-168.
34. NFHS-3: Madhya Pradesh, National Family Health Survey, India. Institute of Population Sciences, Deonar, Mumbai 400008, India: Ministry of Health and Welfare Gol ed.2005-2006;:

35. UNICEF-WHO: Low Birthweight; Country, Regional and Global Estimates. In New York: UNICEF; 2004.

36. Rogerson SJ, Brown HC, Pollina E, Abrams ET, Tadesse E, Lema VM, Molyneux ME: Placental tumor necrosis factor alpha but not gamma interferon is associated with placental malaria and low birth weight in Malawian women. Infect Immun 2003, 71:267-270.

37. Fried M, Muga RO, Misore AO, Duffy PE: Malaria elicits type 1 cytokines in the human placenta: IFN-gamma and TNF-alpha associated with pregnancy outcomes. J Immunol 1998, 160:2523-2530.

38. Moormann AM, Sullivan AD, Rochford RA, Chensue SW, Bock PJ, Nyirenda T, Meshnick SR: Malaria and pregnancy: placental cytokine expression and its relationship to intrauterine growth retardation. J Infect Dis 1999, 180:1987-1993.

39. Abrams ET, Milner DA Jr, Kwiek J, Mwapasa V, Kamwendo DD, Zeng D, Tadesse E, Lema VM, Molyneux ME, Rogerson SJ, Meshnick SR: Risk factors and mechanisms of preterm delivery in Malawi. Am J Reprod Immunol 2004, 52:174-183.

40. Mikolajczyk RT, Zhang J, Betran AP, Souza JP, Mori R, Gulmezoglu AM, Merialdi M: A global reference for fetal-weight and birthweight percentiles. Lancet 2011, 377:1855-1861

41. Galbraith RM, Fox H, Hsi B, Galbraith GM, Bray RS, Faulk WP: The human materno-foetal relationship in malaria. II. Histological, ultrastructural and immunopathological studies of the placenta. Trans $R$ Soc Trop Med Hyg 1980, 74:61-72.

doi:10.1186/1475-2875-13-232

Cite this article as: Ahmed et al:: Placental infections with histologically confirmed Plasmodium falciparum are associated with adverse birth outcomes in India: a cross-sectional study. Malaria Journal 2014 13:232.

\section{Submit your next manuscript to BioMed Central and take full advantage of:}

- Convenient online submission

- Thorough peer review

- No space constraints or color figure charges

- Immediate publication on acceptance

- Inclusion in PubMed, CAS, Scopus and Google Scholar

- Research which is freely available for redistribution 